\title{
Recent update on the prevalence of vibrio species among cultured grouper in Peninsular Malaysia
}

\begin{abstract}
Vibrio infections are common among marine fish and lead to serious problems in the aquaculture sector. This study reports a recent occurrence of Vibrio species (spp.) isolated from cultured groupers in Peninsular Malaysia using the gyrB and pyrH genes. A total of 147 Vibrio strains were successfully isolated from 77 (64\%) groupers using culture method and subjected to gyrB and pyrH sequencing for species identification and confirmation. Results showed that $89 \%$ of Vibrio strains were identified and clustered to six groups of Vibrio spp., while $11 \%$ were not clustered to any Vibrio spp. using the gyrB sequences. Meanwhile, by analysis of the pyrH sequences all the 147 Vibrio strains (100\%) were successfully identified and clustered into 11 groups of Vibrio spp., including the gyrB non-identified strains. The pyrH gene provides a better resolution for identification of Vibrio spp. compared with the gyrB gene. Thus, the pyrH gene was more suitable for a rapid determination of Vibrio spp. distribution in Peninsular Malaysia. Using the pyrH gene, our study found higher prevalence of Vibrio vulnificus (33\%), V. alginolyticus (24\%) and V. parahaemolyticus (22\%), followed by V. rotiferianus $(5 \%)$, V. harveyi $(3 \%)$, V. tubiashii (2\%), V. campbellii (2\%), V. ponticus $(1 \%)$, V. diabolicus (1\%), V. owensii (1\%) and others Vibrio sp. (7\%). Thus, the results of this study revealed that the occurrence of pathogenic vibrios among grouper fish is still high in Malaysian aquaculture. In addition, the pyrH gene was proved as a suitable marker for rapid identification of Vibrio species compared with the gyrB gene.
\end{abstract}

Keyword: Grouper; GyrB; Malaysia; Phylogenetics diversity; PyrH; Vibrio 